\title{
DIFFUSIVE SCATTERING OF HIGH-FREQUENCY PHONONS AT FREE SILICON SURFACES
}

\author{
D. MARX and W. EISENMENGER \\ Universität Stuttgart, Physikalisches Institut, Teilinstitut 1, 7000 Stuttgart 80, Fed. Rep. Germany
}

Received 15 March 1982

Revised manuscript received 7 September 1982

\begin{abstract}
Comparing measurements of high-frequency phonon reflection at the uncovered and optically polished (100)-siliconsurface, with calculations considering phonon focusing, reveal complete diffusive scattering with at most $4 \%$ specular reflection contribution. Two possible mechanisms causing diffusive scattering are discussed.
\end{abstract}

In this letter we discuss the question whether highfrequency acoustic phonons $(\nu \geqslant 280 \mathrm{GHz})$ are scattered specularly or diffusively at a mechanically, chemically or ion-polished free silicon surface. Our experimental arrangement is shown in fig. 1 (insert).

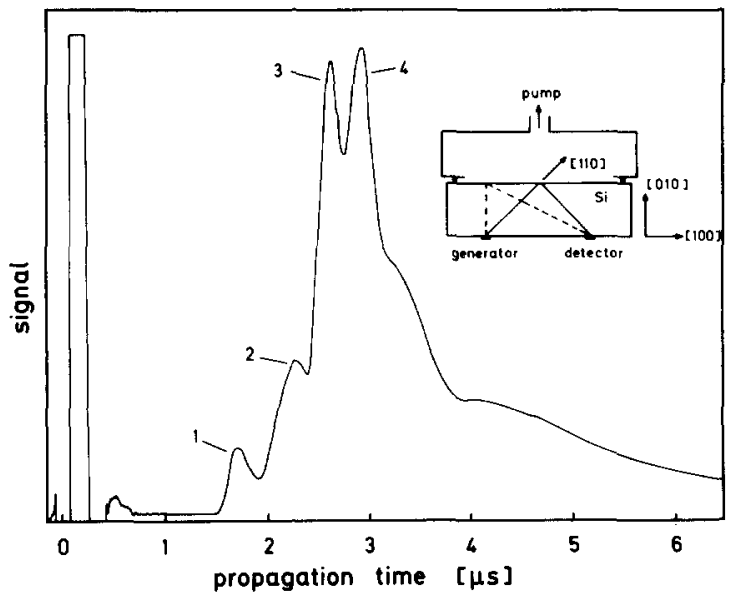

Fig. 1. Typical detector signal for phonons scattered at a free (mechanical, chemical or argon-ion polished) silicon surface. Generator is a constantan film heater, detector a tin-tunneling junction. Pulse duration $100 \mathrm{~ns}$, bath temperature $1.37 \mathrm{~K}$. The pulses are numbered according to their delay time. The inset shows the experimental set-up with the main propagation paths. Pulse no. 1: longitudinal mode propagation along full line. Pulse no. 2: mode converted signal (long $\leftrightarrow$ transv.) propagation path not shown. Pulse no. 3: transverse mode propagation along full line. Pulse no. 4: transverse mode propagation along dotted line (diffuse scattering).
A typical detector signal of the backscattered phonons (fig. 1) consists of four sharp pulses and a broad delayed background. At first sight this may be interpreted as specular reflection from well-defined surface regions with additional diffusive scattering from the entire surface. However, the simple view that a sharp pulse shape indicates specular reflection is misleading since anisotropy of ballistic phonon propagation causes "phonon focusing"; i.e. well-defined directions or channels of phonon propagation. Therefore, even with diffusive scattering at the free surface sharp phonon pulses, i.e. well-defined delay times, can be observed. This is demonstrated by pulse no. 4 propagating with transverse-mode sound velocity along the nonspecular traveling path indicated by the dotted line in the insert of fig. 1. Direct evidence for this traveling path has been given [1] by the significant influence on the phonon-signal amplitude of absorbing layers in small areas directly opposing the phonon generator and detector. From this follows that pulse no. 4 , despite its sharpness, is solely caused by diffuse scat tering.

In contrast to pulse no. 4 the propagation time of pulse no. 3 corresponds to specular reflection of transverse phonons. Since the observed signal amplitude ratio of pulse no. 4 to pulse no. 3 also increases significantly with frequency $[1,2]$, we concluded that pulse no. 3 is caused by specular reflection to a high extent. This reasoning was based on the expected frequency independence of specular reflection (pulse no. 3) and 
the necessary increase with frequency of pure diffuse scattering (pulse no. 4). Therefore, we expected that the signal amplitude of the third pulse, by covering the free silicon surface with a solid metal film (or condensed gas), should be reduced in accordance with the acoustic mismatch theory [3] .

In contrast to this expectation our experiment revealed significant deviations from the acoustic mismatch model. The detected signal amplitude showed about $50 \%$ reflection in strong disagreement with the acoustic model, predicting roughly $1 \%$ reflection for metals and 70-99\% for condensed gases. Therefore, our experiments indicate that the phonon reflection and transmission anomalies at solid-helium interfaces, well known as Kapitza anomaly [4], are also present at solid-solid interfaces. The experimental results depend little on the covering and the preparation of the silicon surface [2]. Influences of the phonon generator and detector properties on the results, such as differences in sensitivity, angular and frequency dependence, could be largely avoided by using a specific comparison technique [2].

From these findings we were led to check our interpretation of the third pulse as a specular one, i.e. to find out whether some focusing channels would result in a sharp signal at the corresponding time delay so that the third pulse is also made up by diffusively scattered phonons.

For this purpose we performed comparative calculations of the total detector pulse signal by the Monte Carlo method, assuming either pure specular or pure diffusive scattering. In these calculations we took full

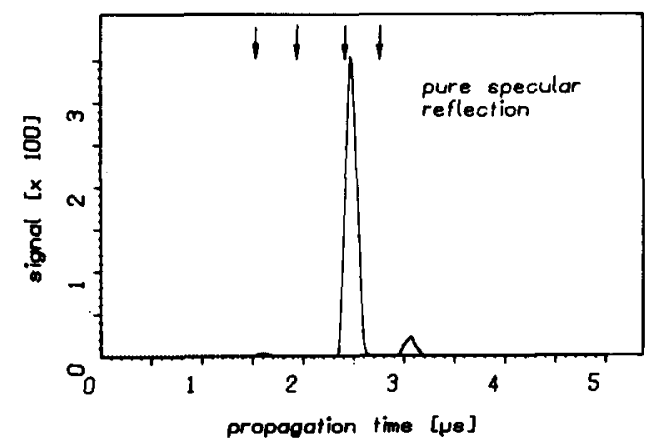

Fig. 2. Calculated detector signal for pure specularly reflected phonons. No mode-converted phonons. Pulse duration 100 ns. The measured propagation times of the pulses are marked by arrows. account of the silicon-crystal anisotropy for phonon propagation (phonon focusing [5]) and the real area and location of generator and detector.

Fig. 2 shows the calculated signal response for pure specular reflection. Mode-converted phonons are not included but the reduction of longitudinal phonons by mode-conversion is taken into account.

As no detailed model of the microscopic diffusive scattering mechanism is known, we assumed the energy of the incident phonons to be distributed on the scattered phonon modes as in quasi-thermal equilibrium (detailed balance). With this assumption we obtained for all possible propagation channels and diffusive scattering the ratio of the scattered phonon intensities in first approximation. Fig. 3 shows the calculated detector signal for pure diffusive scattering, correspondingly.

In comparing fig. 2 and fig. 3 with the experimental result of fig. 1 , we find that the calculated sig. nal amplitudes for pure diffuse scattering agree with the experiment almost completely. Only at maximum $4 \%$ specular reflection can be reconciled with this result. Also the calculated pulse widths for diffusive scattering agree better with the measurements than the calculation for specular reflection. Therefore, our main result is that high-frequency phonons are completely scattered diffusively at uncovered silicon surfaces prepared by conventional procedures such as mechanical, chemical or ion-polishing.

As mentioned, the pulses 1 and 3 , which are due to diffusively scattered phonons with polarization conserved, increase less with phonon frequency than

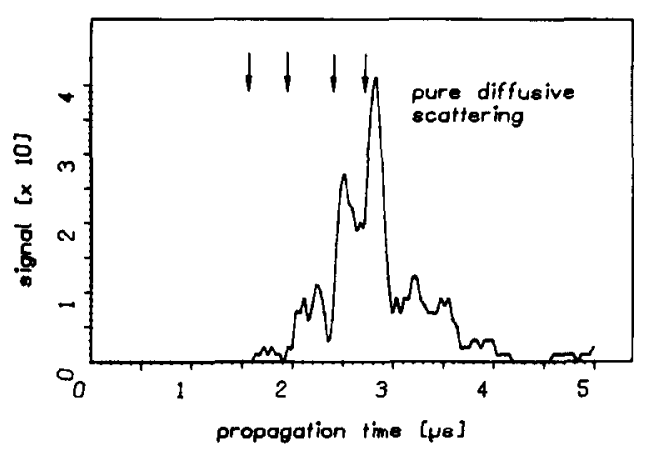

Fig. 3. Calculated detector signal for pure diffusively scattered phonons. Pulse duration 100 ns. The measured propagation times of the pulses are marked by arrows. 
the pulses 2 and 4 where a considerable mode conversion occurs. The different frequency dependence of the pulse-signal amplitudes may be attributed to at least two diffusive scattering mechanisms:

i) The roughness of the silicon surface. The phonon wavelength is small compared with the roughness.

ii) Specific surface states or two-level systems located at the surface [6]. The two-level systems might be formed by adsorbed atoms or molecules.

The mode-conversion in scattering is assumed to be stronger by the second than by the first mechanism. The fraction of phonons scattered by the second mechanism increases with the phonon frequency [6] and gives rise to the frequency dependence of the measured signal.

Since at the free silicon surface high-frequency phonons are not scattered according to the acoustic mismatch theory, one cannot expect that the change of the signal, caused by covering the surface with a solid or with liquid helium, can be described by the mismatch theory. This basically explains the measured discrepancy with respect to the mismatch theory reported earlier [2]. Our calculations show, in addition, that in estimating the fraction of diffusively scattered phonons it is not sufficient to take phonon focusing into account only for the calculation of the propagation time, as Taborek and Goodstein did [7]. It is necessary to consider also the influence of the phonon focusing on the pulse shape.

The microscopic mechanisms of diffusive phonon scattering are presently speculative and are subject to further work.

The authors gratefully acknowledge financial support by the "Deutsche Forschungsgemeinschaft" and valuable discussions with $\mathrm{S}$. Döttinger, $\mathrm{O}$. Koblinger, K. Lassmann and M. Welte.

\section{References}

[1] D. Marx, J. Buck, K. Lassmann and W. Eisenmenger, J. de Phys. C6, Suppl. to No. 8 (1978) C6-1015.

[2] D. Marx and W. Eisenmenger, Phys. Lett. 82A (1981) 291.

[3] W.A. Little, Can. J. Phys. 37 (1959) 334.

[4] A.F.G. Wyatt, in: Nonequilibrium superconductivity, phonons and Kapitza boundaries, ed. K.E. Gray (Plenum Press, New York, 1981).

[5] A.G. Every, Phys. Rev. Lett. 42 (1979) 1065.

[6] H. Kinder, Proc. 16th Intern. Conf. on Low Temp. Phys. (1981) p. 549.

[7] P. Taborek and D.C. Goodstein, Phys. Rev. B22 (1980) 1550 . 